\title{
Can the galactic rotation curves be explained in brane world models?
}

\author{
M. K. Mak* \\ Department of Physics, The University of Hong Kong, Pokfulam Road, Hong Kong, China \\ T. Harko \\ Department of Physics, The University of Hong Kong, Pokfulam Road, Hong Kong, China
}

(Received 19 February 2004; published 30 July 2004)

\begin{abstract}
We consider solutions with conformal symmetry of the static, spherically symmetric gravitational field equations in the vacuum in the brane world scenario. By assuming that the vector field generating the symmetry is nonstatic, the general solution of the field equations on the brane can be obtained in an exact parametric form, with the conformal factor taken as parameter. As a physical application of the obtained solutions we consider the behavior of the angular velocity of a test particle moving in a stable circular orbit. In this case the tangential velocity can be expressed as a function of the conformal factor and some integration constants only. For a specific range of integration constants, the tangential velocity of the test particle tends, in the limit of large radial distances, to a constant value. This behavior is specific to galactic rotation curves and is explained usually by invoking the hypothesis of dark matter. The limiting value of the angular velocity of the test particle can be obtained as a function of the baryonic mass and radius of the galaxy. The behavior of the dark radiation and dark pressure terms is also considered in detail, and it is shown that they can be expressed in terms of the rotational velocity of a test particle. Hence all the predictions of the present model can be tested observationally. Therefore the existence of the nonlocal effects, generated by the free gravitational field of the bulk in a conformally symmetric brane, may provide an explanation for the dynamics of the neutral hydrogen clouds at large distances from the galactic center.
\end{abstract}

DOI: 10.1103/PhysRevD.70.024010 PACS number(s): 04.50. $+\mathrm{h}$, 04.20.Cv, 04.20.Jb, 95.35. $+\mathrm{d}$

\section{INTRODUCTION}

Einstein's theory of general relativity, and some of its generalizations, proved to be in excellent agreement with observational or experimental results in the solar system, binary star systems, or laboratory [1]. However, it has long been known that Newtonian or general relativistic mechanics applied to the visible matter in galaxies and clusters does not correctly describe the dynamics of those systems. The rotation curves of spiral galaxies [2] are one of the best pieces of evidence showing the problems Newtonian mechanics and/or standard general relativity has to face on the galactic or intergalactic scale. In these galaxies neutral hydrogen clouds are observed at large distances from the center, much beyond the extent of the luminous matter. Assuming a nonrelativistic Doppler effect and emission from stable circular orbits in a Newtonian gravitational field, the frequency shifts in the 21$\mathrm{cm}$-line hydrogen emission lines allows measurement of the velocity of the clouds. Since the clouds move in circular orbits with velocity $v_{t g}(r)$, the orbits are maintained by the balance between the centrifugal acceleration $v_{t g}^{2} / r$ and the gravitational attraction force $G M(r) / r^{2}$ of the total mass $M(r)$ contained within the orbit. This allows an expression of the mass profile of the galaxy in the form $M(r)$ $=r v_{t g}^{2} / G$.

Observations show that the rotational velocities increase near the center of the galaxy and then remain nearly constant

\footnotetext{
*Electronic address: mkmak@vtc.edu.hk

†Electronic address: harko@hkucc.hku.hk
}

at a value of $v_{t g \infty} \sim 200 \mathrm{~km} / \mathrm{s}$ [2]. This leads to a mass profile $M(r)=r v_{t g \propto \infty}^{2} / G$. Consequently, the mass within a distance $r$ from the center of the galaxy increases linearly with $r$, even at large distances where very little luminous matter can be detected. This behavior of the galactic rotation curves is explained by postulating the existence of some dark (invisible) matter, distributed in a spherical halo around the galaxies. The dark matter is assumed to be a cold, pressureless medium. There are many possible candidates for dark matter, the most popular ones being weakly interacting massive particles (WIMPs). Their interaction cross sections with normal baryonic matter, while extremely small, are expected to be nonzero and we may expect to detect them directly. It has also been suggested that the dark matter in the Universe might be composed of superheavy particles, with mass $\geqslant 10^{10} \mathrm{GeV}$. But observational results show that the dark matter can be composed of superheavy particles only if these interact weakly with normal matter or if their mass is above $10^{15} \mathrm{GeV}[3]$.

From a general relativistic point of view the space-time geometries associated with dark matter halos were considered in [4], where several properties of this space-time and the characteristics of the possible energy-momentum tensors which could produce such geometries have been discussed. The form of the galactic potentials can be obtained, within a general relativistic framework, from the observed rotation curves, without specific reference to any metric theory of gravity. Given the potential, the gravitational mass can be determined by way of an anisotropy function of the static, spherically symmetric gravitational galactic field [5]. The possibility that dark matter has a substantial amounts of pressure, comparable in magnitude to the energy density, has 
been investigated in [6]. Galaxy halo models, consistent with observations of flat rotation curves, are possible for a variety of equations of state with anisotropic pressures.

However, despite more than 20 years of intense experimental and observational effort, up to now no nongravitational evidence for dark matter has ever been found: no direct evidence of it and no annihilation radiation from it. Moreover, accelerator and reactor experiments do not support the physics (beyond the standard model) on which the dark matter hypothesis is based.

Therefore, it seems that the possibility that Einstein's (and the Newtonian) gravity breaks down at the scale of galaxies cannot be excluded a priori. Several theoretical models, based on a modification of Newton's law or of general relativity, have been proposed to explain the behavior of the galactic rotation curves. A modified gravitational potential of the form $\phi=-G M\left[1+\alpha \exp \left(-r / r_{0}\right)\right] /(1+\alpha) r$, with $\alpha=-0.9$ and $r_{0} \approx 30 \mathrm{kpc}$, can explain flat rotational curves for most of the galaxies [7].

In another model, called Modified Orbital Newtonian Dynamics (MOND), and proposed by Milgrom [8], the Poisson equation for the gravitational potential $\nabla^{2} \phi=4 \pi G \rho$ is replaced by an equation of the form $\nabla\left[\mu(x)\left(|\nabla \phi| / a_{0}\right)\right]$ $=4 \pi G \rho$, where $a_{0}$ is a fixed constant and $\mu(x)$ a function satisfying the conditions $\mu(x)=x$ for $x \ll 1$ and $\mu(x)=1$ for $x \gg 1$. The force law, giving the acceleration $a$ of a test particle, becomes $a=a_{N}$ for $a_{N} \gg a_{0}$ and $a=\sqrt{a_{N} a_{0}}$ for $a_{N}$ $\ll a_{0}$, where $a_{N}$ is the usual Newtonian acceleration. The rotation curves of the galaxies are predicted to be flat, and they can be calculated once the distribution of baryonic matter is known. Alternative theoretical models to explain the galactic rotation curves have been elaborated recently by Mannheim [9] and Moffat and Sokolov [10].

A general analysis of the possibility of an alternative gravity theory explaining the dynamics of galactic systems without dark matter was performed by Zhytnikov and Nester [11]. From very general assumptions about the structure of a relativistic gravity theory (the theory is metric and invariant under general coordinates transformation, has a good linear approximation, it does not possess any unusual gauge freedom, and it is not a higher derivative gravity) a general expression for the metric to order $(v / c)^{2}$ has been derived. This allows us to compare the predictions of the theory with various experimental data: the Newtonian limit, light deflection and retardation, rotation of galaxies, and gravitational lensing. The general conclusion of this study is that the possibility for any gravity theory to explain the behavior of galaxies without dark matter is rather improbable.

The idea that our four-dimensional Universe might be a four-dimensional space-time, embedded in a higher dimensional space-time, had been proposed and studied, from both mathematical and physical points of view, for a long time (for a full account of the existing results on the subject and on the early references see [12]). The embedding approach to gravity has its origins in the book by Eisenhart [13]. The mathematical problems of the embeddings in higher dimensional space-times, with applications to general relativity have been discussed in detail as early as 1965 [14]. By using a more physical approach Akama [15] and Rubakov and
Shaposhnikov [16] have suggested that we may live on a domain wall in a higher dimensional space. Earlier references to these topics can also be found in Bandos and Kummer [17]. In this paper a generalization of the embedding approach for $d$-dimensional gravity based upon $p$-brane theories is considered.

Recently, as a result of the proposal by Randall and Sundrum [18] that our four-dimensional space-time is a threebrane, embedded in a five-dimensional space-time (the bulk), the idea of the embedding of our Universe in a higher dimensional space had attracted again a considerable interest. According to the brane world scenario, the physical fields (electromagnetic, Yang-Mills, etc.) in our four-dimensional Universe are confined to the three-brane. These fields are assumed to arise as fluctuations of branes in string theories. Only gravity can freely propagate in both the brane and bulk space-times, with the gravitational self-couplings not significantly modified. This model originated from the study of a single three-brane embedded in five dimensions, with the 5D metric given by $d s^{2}=e^{-f(y)} \eta_{\mu \nu} d x^{\mu} d x^{\nu}+d y^{2}$, which, due to the appearance of the warp factor, could produce a large hierarchy between the scale of particle physics and gravity. Even if the fifth dimension is uncompactified, standard 4D gravity is reproduced on the brane. Hence this model allows the presence of large or even infinite noncompact extra dimensions. Our brane is identified as a domain wall in a fivedimensional anti-de Sitter space-time. For a review of dynamics and geometry of brane universes see [19].

As a result of the correction terms coming from the extra dimensions, significant deviations from the Einstein theory occur in brane world models at very high energies [20]. Gravity is largely modified at the electroweak scale $1 \mathrm{TeV}$. The cosmological implications of the brane world theories have been extensively investigated in the physical literature [21]. Gravitational collapse can also produce high energies, with five-dimensional effects playing an important role in the formation of black holes [22].

For standard general relativistic spherical compact objects the exterior space-time is described by the Schwarzschild metric. In the five-dimensional brane world models, the high energy corrections to the energy density, together with the Weyl stresses from bulk gravitons, imply that on the brane the exterior metric of a static star is no longer the Schwarzschild metric [23]. The presence of Weyl stresses also means that the matching conditions do not have a unique solution on the brane; knowledge of the five-dimensional Weyl tensor is needed as a minimum condition for uniqueness. Static, spherically symmetric exterior vacuum solutions of the brane world models have been proposed first by Dadhich et al. [23] and Germani and Maartens [24]. The solution obtained in [23] has the mathematical form of the Reissner-Nordström solution, in which a tidal Weyl parameter plays the role of the electric charge of the general relativistic solution. A second exterior solution, which also matches a constant density interior, has been derived in [24]. Other classes of exact or approximate [using the multipole $(1 / r)$ expansion] solutions of vacuum field equations on the brane have been obtained in [25].

The vacuum field equations on the brane have been re- 
duced to a system of two ordinary differential equations, which describe all the geometric properties of the vacuum as functions of the dark pressure and dark radiation terms (the projections of the Weyl curvature of the bulk, generating nonlocal brane stresses) in [26]. Several classes of exact solutions of the vacuum gravitational field equations on the brane have been derived, and vacuums with particular symmetries have been investigated by using Lie group techniques. A homology theorem for the static, spherically symmetric gravitational field equations in the vacuum on the brane has also been proved.

It is the purpose of the present paper to extend the approach initiated in [26] by considering vacuum space-times on the brane that are related to some more general Lie groups of transformations and to investigate their possible physical relevance for the explanation of the dynamics of galaxies. As a group of admissible transformations we chose the oneparameter group of conformal motions. More exactly, we consider spherically symmetric and static solutions of the gravitational field equations for which the metric tensor $g_{\mu \nu}$ has the property $L_{\xi} g_{\mu \nu}=\psi(r) g_{\mu \nu}$, where the left-hand side is the Lie derivative of the metric tensor, describing the gravitational field in vacuum on the brane, with respect to the vector field $\xi^{\mu}$, and $\psi$, the conformal factor, is an arbitrary function of the radial coordinate $r$. As for the vector field $\xi^{\mu}$ we assume that it is nonstatic. With these assumptions the gravitational field equations, describing the static vacuum brane, can be integrated in Schwarzschild coordinates, and an exact solution, corresponding to a brane admitting a oneparameter group of motions, can be obtained. The general solution of the field equations depends on three arbitrary integration constants. The conformal symmetry also uniquely fixes the mathematical form of the dark radiation and dark pressure terms, respectively, which describe the nonlocal effects induced by the gravitational field of the bulk.

As a physical application of the conformally symmetric vacuum brane model we consider the behavior of the angular velocity of a test particle moving in a stable circular orbit. It turns out that for this case the tangential velocity can be expressed as a function of the conformal factor $\psi$ and some constants of integration only, the velocity being inversely proportional to the conformal factor $\psi$. For a specific range of the integration constants the tangential velocity of the test particle tends, in the limit of large radial distances, to a constant value. This behavior is specific to the galactic rotation curves and is explained usually by invoking the hypothesis of dark matter. However, in the present approach the constant velocity in the large $r$ limit of a test particle moving in the gravitational field of a galaxy is due to the existence of nonlocal effects from the bulk, transmitted via the nonzero components of the bulk Weyl tensor and of the conformally symmetric geometrical structure of the static gravitational field on the brane. The existence of the dark radiation term generates an equivalent mass term, which is linearly increasing with distance and is proportional to the baryonic mass of the galaxy. All the relevant physical parameters (metric tensor components, dark radiation, and dark pressure) can be obtained as functions of the tangential velocity, and hence they can be determined observationally.
The present paper is organized as follows. The basic equations describing the spherically symmetric gravitational field equations in the vacuum on the brane are derived in Sec. II. The general solution of the vacuum brane space-times admitting a one-parameter group of conformal motions, with nonstatic conformal symmetry, is obtained in Sec. III. The behavior of the angular velocity of a test particle in stable circular motion is considered in Sec. IV. We conclude and discuss our results in Sec. V.

\section{FIELD EQUATIONS FOR A STATIC, SPHERICALLY SYMMETRIC VACUUM BRANE}

On the five-dimensional space-time (the bulk), with the negative vacuum energy $\Lambda_{5}$ and brane energy momentum as a source of the gravitational field, the Einstein field equations are given by

$$
G_{I J}=k_{5}^{2} T_{I J}, \quad T_{I J}=-\Lambda_{5} g_{I J}+\delta(Y)\left[-\lambda_{b} g_{I J}+T_{I J}^{\text {matter }}\right],
$$

with $\lambda_{b}$ the vacuum energy on the brane and $k_{5}^{2}=8 \pi G_{5}$. In this space-time a brane is a fixed point of $Z_{2}$ symmetry. In the following capital Latin indices run in the range $0, \ldots, 4$, while Greek indices take the values $0, \ldots, 3$.

Assuming a metric of the form $d s^{2}=\left(n_{I} n_{J}\right.$ $\left.+g_{I J}\right) d x^{I} d x^{J}$, with $n_{I} d x^{I}=d \chi$ the unit normal to the $\chi$ $=$ const hypersurfaces and $g_{I J}$ the induced metric on $\chi$ $=$ const hypersurfaces, the effective four-dimensional gravitational equations on the brane (the Gauss equation) take the form [20]:

$$
G_{\mu \nu}=-\Lambda g_{\mu \nu}+k_{4}^{2} T_{\mu \nu}+k_{5}^{4} S_{\mu \nu}-E_{\mu \nu},
$$

where $S_{\mu \nu}$ is the local quadratic energy-momentum correction

$$
S_{\mu \nu}=\frac{1}{12} T T_{\mu \nu}-\frac{1}{4} T_{\mu}{ }^{\alpha} T_{\nu \alpha}+\frac{1}{24} g_{\mu \nu}\left(3 T^{\alpha \beta} T_{\alpha \beta}-T^{2}\right),
$$

and $E_{\mu \nu}$ is the nonlocal effect from the free bulk gravitational field, the transmitted projection of the bulk Weyl tensor $C_{I A J B}, E_{I J}=C_{I A J B} n^{A} n^{B}$, with the property $E_{I J}$ $\rightarrow E_{\mu \nu} \delta_{I}^{\mu} \delta_{J}^{\nu}$ as $\chi \rightarrow 0$. We have also denoted $k_{4}^{2}=8 \pi G$, with $G$ the usual four-dimensional gravitational constant.

The four-dimensional cosmological constant $\Lambda$ and the four-dimensional coupling constant $k_{4}$ are given by $\Lambda$ $=k_{5}^{2}\left(\Lambda_{5}+k_{5}^{2} \lambda_{b}^{2} / 6\right) / 2$ and $k_{4}^{2}=k_{5}^{4} \lambda_{b} / 6$, respectively. In the limit $\lambda_{b}^{-1} \rightarrow 0$ we recover standard general relativity.

The Einstein equation in the bulk and the Codazzi equation also imply conservation of the energy-momentum tensor of the matter on the brane, $D_{\nu} T_{\mu}{ }^{\nu}=0$, where $D_{\nu}$ denotes the brane covariant derivative. Moreover, from the contracted Bianchi identities on the brane it follows that the projected Weyl tensor should obey the constraint $D_{\nu} E_{\mu}{ }^{\nu}=k_{5}^{4} D_{\nu} S_{\mu}{ }^{\nu}$.

The symmetry properties of $E_{\mu \nu}$ imply that in general we can decompose it irreducibly with respect to a chosen fourvelocity field $u^{\mu}$ as [19] 


$$
E_{\mu \nu}=-k^{4}\left[U\left(u_{\mu} u_{\nu}+\frac{1}{3} h_{\mu \nu}\right)+P_{\mu \nu}+2 Q_{(\mu} u_{\nu)}\right],
$$

where $k=k_{5} / k_{4}, h_{\mu \nu}=g_{\mu \nu}+u_{\mu} u_{\nu}$ projects orthogonal to $u^{\mu}$, the "dark radiation" term $U=-k^{4} E_{\mu \nu} u^{\mu} u^{\nu}$ is a scalar, $Q_{\mu}=k^{4} h_{\mu}^{\alpha} E_{\alpha \beta}$ a spatial vector, and $P_{\mu \nu}=-k^{4}\left[h_{(\mu}^{\alpha} h_{\nu)}^{\beta}\right.$ $\left.-\frac{1}{3} h_{\mu \nu} h^{\alpha \beta}\right] E_{\alpha \beta}$ a spatial, symmetric, and trace-free tensor.

In the case of the vacuum state we have $\rho=p=0, T_{\mu \nu}$ $\equiv 0$, and consequently $S_{\mu \nu}=0$. Therefore, by neglecting the effect of the cosmological constant, the field equations describing a static brane take the form

$$
R_{\mu \nu}=-E_{\mu \nu},
$$

with the trace $R$ of the Ricci tensor $R_{\mu \nu}$ satisfying the condition $R=R_{\mu}^{\mu}=E_{\mu}^{\mu}=0$.

In the vacuum case $E_{\mu \nu}$ satisfies the constraint $D_{\nu} E_{\mu}{ }^{\nu}$ $=0$. In an inertial frame at any point on the brane we have $u^{\mu}=\delta_{0}^{\mu}$ and $h_{\mu \nu}=\operatorname{diag}(0,1,1,1)$. In a static vacuum $Q_{\mu}=0$ and the constraint for $E_{\mu \nu}$ takes the form [24]

$$
\frac{1}{3} D_{\mu} U+\frac{4}{3} U A_{\mu}+D^{\nu} P_{\mu \nu}+A^{\nu} P_{\mu \nu}=0
$$

where $D_{\mu}$ is the projection (orthogonal to $u^{\mu}$ ) of the covariant derivative and $A_{\mu}=u^{\nu} D_{\nu} u_{\mu}$ is the four-acceleration. In the static spherically symmetric case we may choose $A_{\mu}$ $=A(r) r_{\mu}$ and $P_{\mu \nu}=P(r)\left(r_{\mu} r_{\nu}-\frac{1}{3} h_{\mu \nu}\right)$, where $A(r)$ and $P(r)$ (the "dark pressure") are some scalar functions of the radial distance $r$, and $r_{\mu}$ is a unit radial vector [23].

We chose the static spherically symmetric metric on the brane in the form

$$
d s^{2}=-e^{\nu(r)} d t^{2}+e^{\lambda(r)} d r^{2}+r^{2}\left(d \theta^{2}+\sin ^{2} \theta d \phi^{2}\right) .
$$

Then the gravitational field equations and the effective energy-momentum tensor conservation equation in the vacuum take the form [26]

$$
\begin{aligned}
& -e^{-\lambda}\left(\frac{1}{r^{2}}-\frac{\lambda^{\prime}}{r}\right)+\frac{1}{r^{2}}=\frac{48 \pi G}{k^{4} \lambda_{b}} U \\
& e^{-\lambda}\left(\frac{\nu^{\prime}}{r}+\frac{1}{r^{2}}\right)-\frac{1}{r^{2}}=\frac{16 \pi G}{k^{4} \lambda_{b}}(U+2 P), \\
& e^{-\lambda}\left(\nu^{\prime \prime}+\frac{\nu^{\prime 2}}{2}+\frac{\nu^{\prime}-\lambda^{\prime}}{r}-\frac{\nu^{\prime} \lambda^{\prime}}{2}\right)=\frac{32 \pi G}{k^{4} \lambda_{b}}(U-P), \\
& \nu^{\prime}=-\frac{U^{\prime}+2 P^{\prime}}{2 U+P}-\frac{6 P}{r(2 U+P)} .
\end{aligned}
$$

In the following we shall denote $\alpha=16 \pi G / k^{4} \lambda_{b}$.

\section{GENERAL SOLUTION OF THE VACUUM BRANE FIELD EQUATIONS WITH NONSTATIC CONFORMAL SYMMETRY}

The system of the field equations for the vacuum on the brane is underdetermined. A functional relation between the dark energy $U$ and the dark pressure $P$ must be specified in order to solve the equations. An alternative method, which avoids ad hoc specifications, is to assume that the brane is mapped conformally onto itself along the direction $\xi$, so that

$$
L_{\xi} g_{\mu \nu}=g_{\mu \nu, \lambda} \xi^{\lambda}+g_{\lambda \nu} \xi_{, \mu}^{\lambda}+g_{\mu \lambda} \xi_{, \nu}^{\lambda}=\psi g_{\mu \nu},
$$

where $\psi$ is the conformal factor. As for the choice of $\xi$, Herrera and Ponce de Leon [27] assumed that

$$
\boldsymbol{\xi}=\xi^{0}(r) \frac{\partial}{\partial t}+\xi^{1}(r) \frac{\partial}{\partial r}
$$

Using this form of the conformal vector in Eqs. (12) one obtains $\quad \xi^{0}=A, \quad \xi^{1}=(B / 2) r \exp (-\lambda / 2), \quad \psi(r)$ $=B \exp (-\lambda / 2)$, and $\exp (\nu)=C^{2} r^{2}$, where $A, B, C$ are constants. $A$ may be set to zero since $A \partial / \partial t$ is a Killing vector and $B$ may be set to 1 by a rescaling $\xi \rightarrow B^{-1} \xi, \psi \rightarrow B^{-1} \psi$, which leaves Eq. (12) invariant. This form of $\xi$ gives the most general $\xi$ invariant under the Killing symmetries-that is, $[\partial / \partial t, \xi]=0=\left[\mathbf{X}_{\alpha}, \xi\right]$, where $\mathbf{X}_{\alpha}$ generates $S O(3)$. This form of the metric, obtained by imposing static conformal symmetry, has been used in [28] to investigate the properties of strange stars. The general solution of the vacuum brane gravitational field equations for this choice of $\xi$ has been obtained in [26].

A more general conformal symmetry has been proposed by Maartens and Maharajah [29], which generalizes the isotropic conformal vector $t \partial / \partial t+r \partial / \partial r$ of the Minkowski space-time, but weakens the static symmetry of $\xi$ in Eq. (13):

$$
\xi=\xi^{0}(t, r) \frac{\partial}{\partial t}+\xi^{1}(t, r) \frac{\partial}{\partial r} .
$$

Moreover, we assume that the conformal factor $\psi$ is static, $\psi=\psi(r)$. With this form of $\xi$, Eq. (12) gives, immediately (we denote ${ }^{\prime}=d / d r$ ),

$$
\begin{gathered}
\nu^{\prime} \xi^{1}+2 \frac{\partial \xi^{0}}{\partial t}=\psi, \\
\lambda^{\prime} \xi^{1}+2 \frac{\partial \xi^{1}}{\partial r}=\psi, \\
\xi^{1}=\frac{r \psi}{2} .
\end{gathered}
$$

By solving Eqs. (15)-(17) we obtain [29]

$$
\begin{gathered}
\xi^{0}=A+\frac{1}{2} \frac{k}{B} t, \\
\psi=B e^{-\lambda / 2},
\end{gathered}
$$




$$
e^{\nu}=C^{2} r^{2} \exp \left(-2 k B^{-1} \int \frac{d r}{r \psi}\right)
$$

where $k$ is a separation constant and $A, B$, and $C$ are integration constants. Without any loss of generality we can choose $A=0$. Thus for the vector field $\boldsymbol{\xi}$ we obtain

$$
\xi=\frac{1}{2} \frac{k}{B} t \frac{\partial}{\partial t}+\frac{r \psi(r)}{2} \frac{\partial}{\partial r}
$$

while the metric tensor components of the static vacuum brane can be expressed as a function of the conformal factor in the form $\exp (\lambda)=B^{2} \psi^{-2}$ and $\exp (\nu)$ $=C^{2} r^{2} \exp \left(-2 k B^{-1} \int d r / r \psi\right)$, respectively.

Substitution of these forms of the metric functions in the field equations (8)-(10) gives

$$
\begin{gathered}
-\frac{\psi^{2}}{B^{2}}\left(\frac{1}{r^{2}}+\frac{2}{r} \frac{\psi^{\prime}}{\psi}\right)+\frac{1}{r^{2}}=3 \alpha U, \\
\frac{\psi^{2}}{B^{2}}\left(\frac{3}{r^{2}}-2 \frac{k}{B} \frac{1}{r^{2} \psi}\right)-\frac{1}{r^{2}}=\alpha(U+2 P), \\
\psi^{2}\left(2 \frac{\psi^{\prime}}{r \psi}-2 \frac{k}{B} \frac{1}{r^{2} \psi}+\frac{k^{2}}{B^{2}} \frac{1}{r^{2} \psi^{2}}+\frac{1}{r^{2}}\right)=\alpha(U-P) .
\end{gathered}
$$

By multiplying Eq. (24) by 2, adding the equation thus obtained to Eq. (23), and equating the resulting equation with Eq. (22) gives the following differential equation satisfied by the function $\psi$ :

$$
3 r \psi \psi^{\prime}+3 \psi^{2}-3 \frac{k}{B} \psi+\frac{k^{2}}{B^{2}}-B^{2}=0
$$

For $k \neq \pm B^{2}$ the general solution of Eq. (25) is given by

$$
r^{2}=R_{0}^{2} \frac{F(\psi)}{\left|3 \psi^{2}-3 \frac{k}{B} \psi+\frac{k^{2}}{B^{2}}-B^{2}\right|},
$$

where $R_{0}$ is an arbitrary constant of integration:

$$
F(\psi)=\exp \left(-3 \frac{k}{B} \int \frac{d \psi}{3 \psi^{2}-3 \frac{k}{B} \psi+\frac{k^{2}}{B^{2}}-B^{2}}\right)
$$

and

$$
\begin{gathered}
F(\psi)=\left(\frac{\left|\psi-\psi_{2}\right|}{\left|\psi-\psi_{1}\right|}\right)^{m}, \quad k \in\left(-2 B^{2}, 2 B^{2}\right), \\
F(\psi)=\exp \left(\frac{ \pm 2 B}{\psi \mp B}\right), \quad k= \pm 2 B^{2}, \\
F(\psi)=\exp \left[-\frac{k}{B} n \arctan n\left(\psi-\frac{k}{2 B}\right)\right], \\
k \in\left(-\infty,-2 B^{2}\right) \cup\left(2 B^{2},+\infty\right) .
\end{gathered}
$$

In Eqs. (28)-(30) we have also denoted

$$
\begin{gathered}
\psi_{1,2}=\frac{3 \frac{k}{B} \pm \sqrt{12 B^{2}-3 \frac{k^{2}}{B^{2}}}}{6}, \\
m=\frac{3 k}{B \sqrt{12 B^{2}-3 \frac{k^{2}}{B^{2}}}}, \quad n=\frac{6}{\sqrt{3 \frac{k^{2}}{B^{2}}-12 B^{2}}} .
\end{gathered}
$$

For the dark radiation and dark pressure we obtain the general expressions

$$
U(\psi)=\frac{\left|3 \psi^{2}-3 \frac{k}{B} \psi+\frac{k^{2}}{B^{2}}-B^{2}\right|\left(\psi^{2}-2 \frac{k}{B} \psi+\frac{2}{3} \frac{k^{2}}{B^{2}}+\frac{1}{3} B^{2}\right)}{3 \alpha B^{2} R_{0}^{2} F(\psi)}
$$

and

$$
P(\psi)=\frac{\left|3 \psi^{2}-3 \frac{k}{B} \psi+\frac{k^{2}}{B^{2}}-B^{2}\right|\left[4 \psi^{2}-2 \frac{k}{B} \psi-\frac{1}{3}\left(\frac{k^{2}}{B^{2}}-B^{2}\right)-2\right]}{3 \alpha R_{0}^{2} F(\psi)}
$$

respectively.

Generally, $\psi$ cannot be expressed in an exact analytical form as a function of $r$. Hence the functions $\exp (\lambda)=B^{2} \psi^{-2}$, 


$$
\exp (\nu)=\frac{C^{2} R_{0}^{2}}{F(\psi)\left(3 \psi^{2}-3 \frac{k}{B} \psi+\frac{k^{2}}{B^{2}}-B^{2}\right)}
$$

$U(\psi)$ and $P(\psi)$ can be obtained, as functions of the radial distance $r$, only in a parametric form, with $\psi$ taken as parameter.

However, because of the arbitrariness in the choice of the reference system in the general theory of relativity, we can subject the coordinates to any transformation which does not violate the central symmetry of the line element. Therefore, by introducing a new radial coordinate $\bar{r}=\psi(r)$, so that

$$
r=r(\bar{r})=\frac{R_{0} \sqrt{F(\bar{r})}}{\sqrt{\left|3 \bar{r}^{2}-3 \frac{k}{B} \bar{r}+\frac{k^{2}}{B^{2}}-B^{2}\right|}},
$$

we obtain the line element of the static, spherically symmetric metric admitting a conformal symmetry with a nonstatic vector field on the vacuum brane in the form

$$
d s^{2}=\frac{R_{0}^{2}}{F(\bar{r})\left|3 \bar{r}^{2}-3 \frac{k}{B} \bar{r}+\frac{k^{2}}{B^{2}}-B^{2}\right|}\left[-C^{2} d t^{2}+\frac{9 B^{2} F^{2}(\bar{r}) d \bar{r}^{2}}{\left|3 \bar{r}^{2}-3 \frac{k}{B} \bar{r}+\frac{k^{2}}{B^{2}}-B^{2}\right|^{2}}+F^{2}(\bar{r}) d \Omega^{2}\right], \quad k \neq \pm B^{2},
$$

where $d \Omega^{2}=d \theta^{2}+\sin ^{2} \theta d \phi^{2}$ is the metric of a unit sphere.

Therefore, by using the new variable $\bar{r}$ the three classes of conformally symmetric solutions of the gravitational field equations on the brane take the form

$$
\begin{aligned}
d s^{2}= & \frac{R_{0}^{2}}{3} \frac{\left|\bar{r}-\psi_{1}\right|^{m-1}}{\left|\bar{r}-\psi_{2}\right|^{m+1}}\left[-C^{2} d t^{2}+B^{2} \frac{\left|\bar{r}-\psi_{2}\right|^{2 m-2}}{\left|\bar{r}-\psi_{1}\right|^{2 m+2}} d \bar{r}^{2}+\frac{\left|\bar{r}-\psi_{2}\right|^{2 m}}{\left|\bar{r}-\psi_{1}\right|^{2 m}} d \Omega^{2}\right], \quad k \in\left(-2 B^{2}, 2 B^{2}\right), \\
d s^{2}= & \frac{R_{0}^{2}}{3} \frac{\exp \left(\frac{\bar{\mp} B}{\bar{r} \mp B}\right)}{(\bar{r} \mp B)^{2}}\left[-C^{2} d t^{2}+B^{2} \frac{\exp \left(\frac{ \pm 4 B}{\bar{r} \mp B}\right)}{(\bar{r} \mp B)^{4}} d \bar{r}^{2}+\exp \left(\frac{ \pm 4 B}{\bar{r} \mp B}\right) d \Omega^{2}\right], \quad k= \pm 2 B^{2}, \\
d s^{2}= & \frac{R_{0}^{2} \exp \left[\frac{k}{B} n \arctan n\left(\bar{r}-\frac{k}{2 B}\right)\right]}{\left|3 \bar{r}^{2}-3 \frac{k}{B} \bar{r}+\frac{k^{2}}{B^{2}}-B^{2}\right|}\left\{-C^{2} d t^{2}+\frac{9 B^{2} \exp \left[-2 \frac{k}{B} n \arctan n\left(\bar{r}-\frac{k}{B}\right)\right] d \bar{r}^{2}}{B^{2}-B^{2} \mid} \mid\right. \\
& \left.+\exp \left[-2 \frac{k}{B} n \arctan n\left(\bar{r}-\frac{k}{2 B}\right)\right] d \Omega^{2}\right\}, \quad k \in\left(-\infty,-2 B^{2}\right) \cup\left(2 B^{2},+\infty\right) .
\end{aligned}
$$

The general solution of the field equations can be obtained in an exact analytical form for some particular values of $k$. Hence by taking $k= \pm B^{2}$ we immediately obtain

$$
\psi=\frac{R_{0}}{r} \pm B,
$$

with the corresponding line element given by 


$$
d s^{2}=\frac{1}{\left(\frac{R_{0}}{r} \pm B\right)^{2}}\left(-C^{2} d t^{2}+B^{2} d r^{2}\right)+r^{2}\left(d \theta^{2}+\sin ^{2} \theta d \phi^{2}\right), \quad k= \pm B^{2} .
$$

For the dark radiation and the dark pressure we find

$$
U(r)=\frac{1}{\alpha R_{0}^{2}}\left(\frac{R_{0}}{r}\right)^{4}, \quad P(r)=\frac{2}{\alpha R_{0}^{2}}\left(\frac{2 R_{0}}{3 r} \pm B\right)\left(\frac{R_{0}}{r}\right)^{3}, \quad k= \pm B^{2} .
$$

\section{STABLE CIRCULAR ORBITS IN CONFORMALLY SYMMETRIC SPACE-TIMES ON THE BRANE}

We shall consider now the problem of constructing stable circular timelike geodesic orbits in a static spherically, spherically symmetric field on the brane, with line element given in a general form by Eq. (7). The motion of a test particle in the gravitational field can be described by the Lagrangian [4]

$$
2 L=\left(\frac{d s}{d \tau}\right)^{2}=-e^{\nu(r)}\left(\frac{d t}{d \tau}\right)^{2}+e^{\lambda(r)}\left(\frac{d r}{d \tau}\right)^{2}+r^{2}\left(\frac{d \Omega}{d \tau}\right)^{2},
$$

where we denoted by $\tau$ the affine parameter along the geodesics. In the timelike case $\tau$ corresponds to the proper time. In the following we denote by an overdot the differentiation with respect to $\tau$. From the Lagrangian given by Eq. (43) it follows that the energy $E=e^{\nu} \dot{t}$ and the $\varphi$ component $l_{\varphi}$ $=r^{2} \sin ^{2} \theta \dot{\varphi}$ of the angular momentum of the particle are conserved quantities, $E=$ const and $l_{\varphi}=$ const. The $\theta$ component of the angular momentum, $l_{\theta}=r^{2} \dot{\theta}$, is not a constant of motion, but the total angular momentum $l^{2}=l_{\theta}^{2}+\left(l_{\varphi} / \sin \theta\right)^{2}$ is a conserved quantity, $l^{2}=$ const. The total angular momentum can be expressed in terms of the solid angle as $l^{2}=r^{4} \dot{\Omega}^{2}$ [4].

In the timelike case the equation of the geodesic orbits can be written in the form

$$
\dot{r}^{2}+V(r)=0,
$$

where the potential $V(r)$ is given by

$$
V(r)=-e^{-\lambda}\left(E^{2} e^{-\nu}-\frac{l^{2}}{r^{2}}-1\right) .
$$

Restricting the radial motion to stable circular orbits implies imposing the conditions $\dot{r}=0$ and $\partial V / \partial r=0$, so that the potential describes an extremum of the motion. In order that this extremum be a minimum the condition $\partial^{2} V / \partial r^{2}$ $>0$ is also required. These three conditions imply that the circular motion is stable. They also lead to the following expressions of the energy and total angular momentum of the particle [4,5]:

$$
E^{2}=\frac{2 e^{\nu}}{2-r \nu^{\prime}}, \quad l^{2}=\frac{r^{3} \nu^{\prime}}{2-r \nu^{\prime}} .
$$

On the other hand, the line element, given by Eq. (7), can be rewritten in terms of the spatial components of the velocity, normalized with the speed of light, measured by an inertial observer far from the source, as $d s^{2}=-d t^{2}\left(1-v^{2}\right)$ [4], where

$$
v^{2}=e^{-v}\left[e^{\lambda}\left(\frac{d r}{d t}\right)^{2}+r^{2}\left(\frac{d \Omega}{d t}\right)^{2}\right]
$$

For a stable circular orbit, $\dot{r}=0$, and the tangential velocity of the test particle can be expressed as

$$
v_{t g}^{2}=\frac{r^{2}}{e^{\nu}}\left(\frac{d \Omega}{d t}\right)^{2} .
$$

In terms of the conserved quantities the angular velocity is given by

$$
v_{t g}^{2}=\frac{e^{\nu}}{r^{2}} \frac{l^{2}}{E^{2}}
$$

With the use of Eqs. (46) we obtain

$$
v_{t g}^{2}=\frac{r \nu^{\prime}}{2} .
$$

Thus, the rotational velocity of the test body is determined by the metric coefficient $\exp (\nu)$ only.

In the case of the motion of a test particle in a conformally symmetric, static spherically symmetric space-time, with a nonstatic vector field generating the symmetry, the metric coefficient $\exp (\nu)$ is given by Eq. (20). Therefore for the angular velocity we find the simple expression

$$
v_{t g}^{2}=1-\frac{k}{B} \frac{1}{\psi} .
$$

Equation (51) gives a simple physical interpretation of the conformal factor $\psi$ in terms of the tangential velocity, $\psi$ $=(k / B)\left[\left(1-v_{t g}^{2}\right)^{-1}\right]$. On the other hand, the metric coefficient $\exp (\lambda)$ can also be expressed as a function of the tangential velocity only:

$$
\exp (\lambda)=\frac{B^{4}}{k^{2}}\left(1-v_{t g}^{2}\right)^{2}
$$




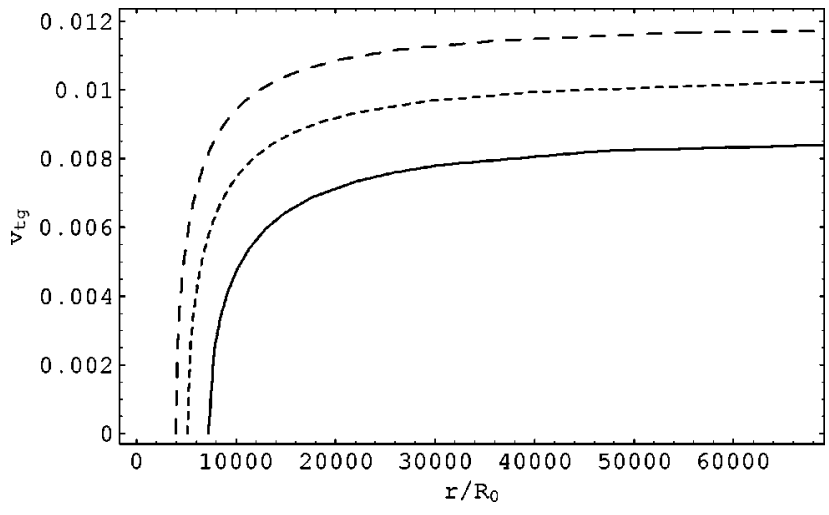

FIG. 1. Variation, as a function of the parameter $r / R_{0}$, of the tangential velocity $v_{t g}$ of a test particle in a stable circular orbit in a conformally symmetric vacuum space-time on the brane, for $B$ $=1.00001$ and different values of $k: k=0.9999$ (solid curve), $k$ $=0.99985$ (dotted curve), and $k=0.9998$ (dashed curve).

From Eq. (51) it follows that the general, physically acceptable, range of the parameter $\psi$ is $\psi \in[k / B, \infty)$, corresponding to a variation of the tangential velocity between zero and the speed of light. However, in the case $k \in\left(-2 B^{2}, 2 B^{2}\right)$, the limiting value of the radial coordinate, $r \rightarrow \infty$, is obtained, as one can see from Eq. (28), in the limits $\psi \rightarrow \psi_{1}$ or $\psi \rightarrow \psi_{2}$ (the corresponding limit depends on the numerical values of the parameters $k$ and $B$ ). Assuming that $r \rightarrow \infty$ for $\psi \rightarrow \psi_{1}$, it follows that in the large $r$ limit the tangential velocity of a test particle in stable circular motion in a conformally symmetric static vacuum space-time on the brane tends to a limiting, nonzero value $v_{\operatorname{tg} \infty}, v \rightarrow v_{t g \infty}, r$ $\rightarrow \infty$, given by

$$
v_{\operatorname{tg} \infty}=\sqrt{1-\frac{6 k}{3 k+\sqrt{12 B^{4}-3 k^{2}}}} .
$$

For $B=1.00000034$ and $k=0.9999999$ the limiting tangential velocity is given by $v_{t g \infty} \sim 0.00072 \sim 216.3 \mathrm{~km} / \mathrm{s}$, which is of the order of the observed galactic rotational velocities.

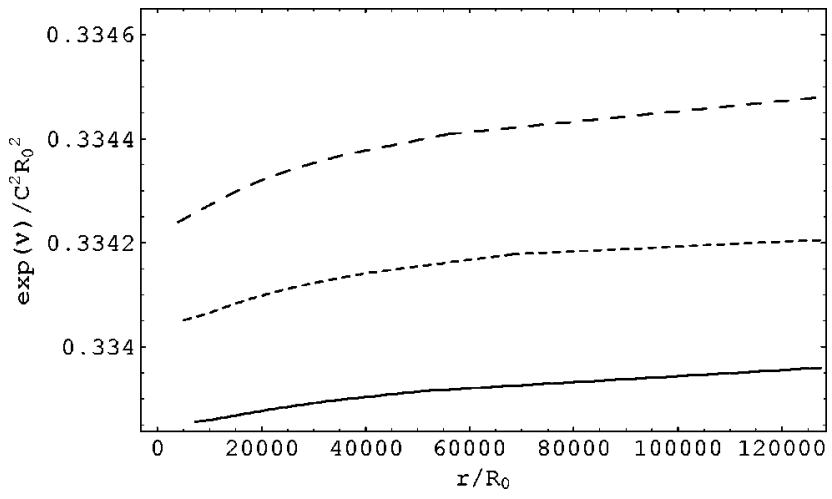

FIG. 2. Variation, as a function of the parameter $r / R_{0}$, of the metric coefficient $\exp (\nu) / C^{2} R_{0}^{2}$ for a static, conformally symmetric vacuum space-time on the brane, for $B=1.00001$ and different values of $k: k=0.9999$ (solid curve), $k=0.99985$ (dotted curve), and $k=0.9998$ (dashed curve).

In the case of a conformally symmetric static vacuum space-time on the brane, the general dependence of the tangential velocity $v_{t g}$ on the radial coordinate $r$ is given, with the use of Eq. (26), in a parametric form, with $\psi$ taken as parameter. In this model it is not possible to express the tangential velocity as an analytical function of $r$.

The variation of $v_{t g}$ as a function of the radial distance is represented, for some particular values of $k$ and $B$, in Fig. 1 .

In the limit of large $r, r \rightarrow \infty$, and for this choice of the numerical values of the arbitrary parameters $k$ and $B$, the tangential velocity tends to a constant value. The numerical value of the limiting velocity is extremely sensitive to the values of $k$ and $B$.

The variations of the metric coefficients $\exp (\nu)$ and $\exp (\lambda)$ are represented in Figs. 2 and 3, respectively.

The metric components satisfy the conditions $\exp (\nu) \leqslant 1$ and $\exp (\lambda) \geqslant 1$, respectively.

The dark radiation and dark pressure terms can also be represented, as a function of the tangential velocity of a test body, in the form

$$
U\left(v_{t g}\right)=\frac{\left\{B^{4}\left(1-v_{t g}^{2}\right)+k^{2}\left[2 v_{t g}^{2}\left(1+v_{t g}^{2}\right)-1\right]\right\}\left|k^{2}\left[1+v_{t g}^{2}\left(1+v_{t g}^{2}\right)\right]-B^{4}\left(1-v_{t g}^{2}\right)^{2}\right|}{9 \alpha R_{0}^{2} B^{6}\left(1-v_{t g}^{2}\right)^{4} F\left[\frac{k}{B}\left(1-v_{t g}^{2}\right)^{-1}\right]}
$$

and

$$
P\left(v_{t g}\right)=\frac{\left\{B^{2}\left(B^{2}-6\right)\left(1+v_{t g}^{2}\right)+k^{2}\left[5+v_{t g}^{2}\left(8-v_{t g}^{2}\right)\right]\right\}\left|k^{2}\left[1+v_{t g}^{2}\left(1+v_{t g}^{2}\right)\right]-B^{4}\left(1-v_{t g}^{2}\right)^{2}\right|}{9 \alpha R_{0}^{2} B^{4} F\left[\frac{k}{B}\left(1-v_{t g}^{2}\right)^{-1}\right]},
$$




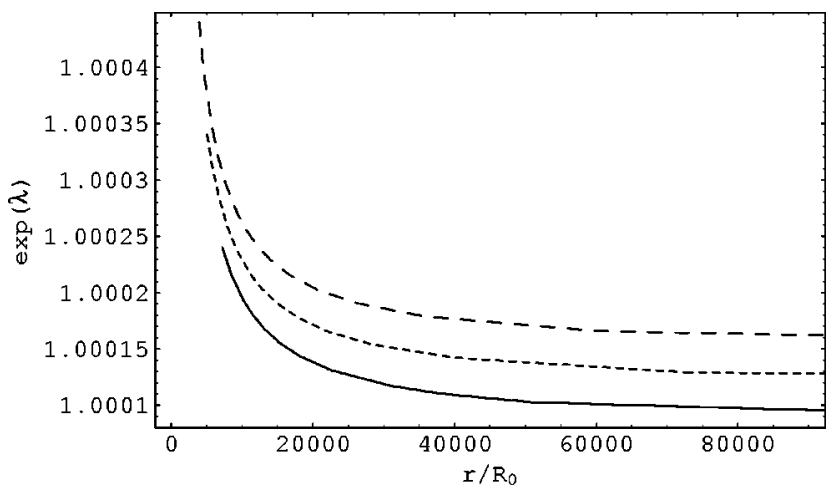

FIG. 3. Variation, as a function of the parameter $r / R_{0}$, of the metric coefficient $\exp (\lambda)$ for a static, conformally symmetric vacuum space-time on the brane, for $B=1.00001$ and different values of $k$ : $k=0.9999$ (solid curve), $k=0.99985$ (dotted curve), and $k=0.9998$ (dashed curve).

respectively.

The variation of the dark radiation $U$ is represented, as a function of $r / R_{0}$, in Fig. 4.

The dark radiation term is positive for all values of the radial coordinate $r, U(r) \geqslant 0, \forall r \in(0, \infty)$. In the limit of large $r, U$ tends to zero, $\lim _{r \rightarrow \infty} U(r)=0$. The variation of the dark pressure as a function of $r$ is represented in Fig. 5.

In the present model the dark pressure is negative, satisfying the condition $P(r) \leqslant 0, \forall r \in(0, \infty)$. In the large time limit, similar to the dark radiation term, the dark pressure also tends to zero, $\lim _{r \rightarrow \infty} P(r)=0$.

\section{DISCUSSIONS AND FINAL REMARKS}

In order to obtain a manifestly coordinate invariant characterization of certain geometrical properties of geometries, like, for example, curvature singularities, Petrov type of the Weyl tensor, etc., the scalar invariants of the Riemann tensor have been extensively used. Two scalars, which have been considered in the physical literature, are the Kretschmann scalars, RiemSq $\equiv R_{i j k l} R^{i j k l}$ and $\mathrm{RicciSq} \equiv R_{i j} R^{i j}$, where $R_{i j k l}$ is the Riemann curvature tensor.

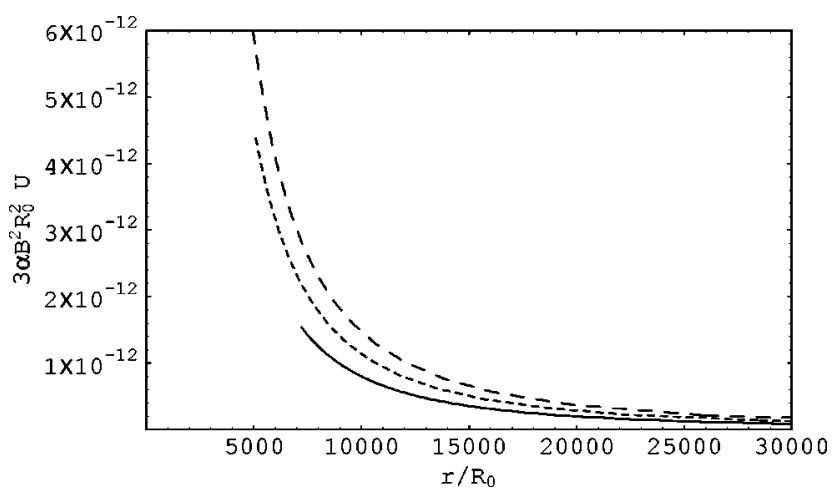

FIG. 4. Variation, as a function of the parameter $r / R_{0}$, of the dark radiation term $3 \alpha B^{2} R_{0}^{2} U$ for a static, conformally symmetric vacuum space-time on the brane, for $B=1.00001$ and different values of $k: k=0.9999$ (solid curve), $k=0.99985$ (dotted curve), and $k=0.9998$ (dashed curve).

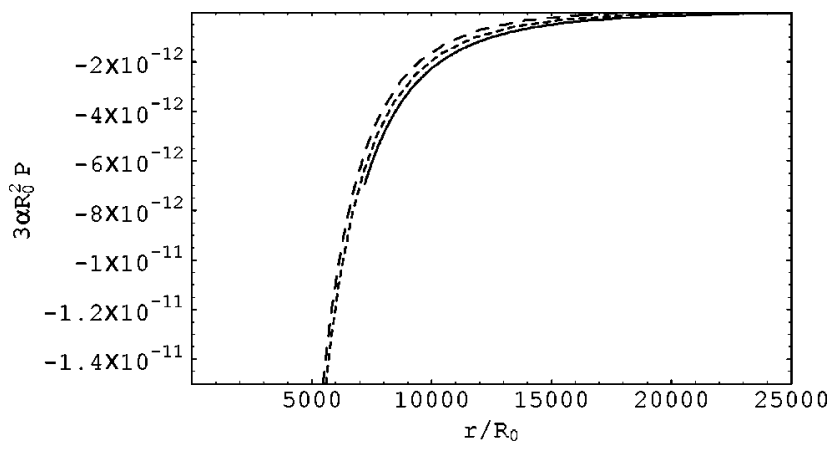

FIG. 5. Variation, as a function of the parameter $r / R_{0}$, of the dark pressure term $3 \alpha R_{0}^{2} P$ for a static, conformally symmetric vacuum space-time on the brane, for $B=1.00001$ and different values of $k: k=0.9999$ (solid curve), $k=0.99985$ (dotted curve), and $k=0.9998$ (dashed curve).

For space-times which are the product of 2 twodimensional spaces, one Lorentzian and one Riemannian, subject to a separability condition on the function which couples the two-spaces, it has been suggested in [30] that the set $C=\left\{R, r_{1}, r_{2}, w_{2}\right\}$ forms an independent set of scalar polynomial invariants, satisfying the number of degrees of freedom in the curvature. $R=g^{i l} g^{j k} R_{i j k l}$ is the Ricci scalar and the quantities $r_{1}, r_{2}$, and $w_{2}$ are defined according to [31]

$$
\begin{aligned}
r_{1}= & \phi_{A B \dot{A} \dot{B}} \phi^{A B \dot{A} \dot{B}}=\frac{1}{4} S_{a}^{b} S_{b}^{a}, \\
r_{2}= & \phi_{A B \dot{A} \dot{B}} \phi_{C \dot{C}}^{B \dot{B}} \phi^{C A \dot{C} \dot{A}}=-\frac{1}{8} S_{a}^{b} S_{b}^{c} S_{c}^{a}, \\
w_{2}= & \Psi_{A B C D} \Psi_{E F}^{C D} \Psi^{E F A B} \\
= & \frac{1}{32}\left(3 E_{b}^{a} H_{c}^{b} H_{a}^{c}-E_{b}^{a} E_{c}^{b} E_{a}^{c}\right) \\
& +\frac{i}{32}\left(H_{b}^{a} H_{c}^{b} H_{a}^{c}-3 E_{b}^{a} E_{c}^{b} H_{a}^{c}\right),
\end{aligned}
$$

where $S_{a}^{b}=R_{a}^{b}-\frac{1}{4} R \delta_{a}^{b}$ is the trace-free Ricci tensor, $\phi_{A B \dot{A} \dot{B}}$ denotes the spinor equivalent of $S_{a b}, \Psi_{A B C D}$ denotes the spinor equivalent of the Weyl tensor $C_{a b c d}$, and $\bar{C}_{a b c d}$ denotes the complex conjugate of the self-dual Weyl tensor, $C_{a b c d}^{+}=\frac{1}{2}\left(C_{a b c d}-i^{*} C_{a b c d}\right) . \quad E_{a c}=C_{a b c d} u^{b} u^{d}$ and $H_{a c}$ $=C_{a b c d}^{*} u^{b} u^{d}$ are the "electric" and "magnetic" parts of the Weyl tensor, respectively, where $u^{a}$ is a timelike unit vector and $C_{a b c d}^{*}=\frac{1}{2} \eta_{a b e f} C_{c d}^{e f}$ is the dual tensor. The expressions of the invariants for some particular values of the integration constants $k$ and $B$ are presented in the Appendix. As a result of their complicated form, we shall not present the values of the invariants for other values of $k$ and $B$. For the $B=1, k$ $=2$ case the invariants diverge at $r=1$, while for the $B$ $=1, k= \pm 1$ case they diverge for $r \rightarrow 0$.

In the present paper we have obtained all the conformally symmetric solutions of the vacuum field equations in the brane world model, under the assumption of a nonstatic conformal symmetry, and we have discussed some of their physical properties. In particular we have considered the be- 
havior of the angular velocity of a test particle in a stable circular orbit on the brane. The conformal factor $\psi$, together with two constants of integration, uniquely determines the rotational velocity of the particle. In the limit of large radial distances and for a particular set of values of the integration constants the angular velocity tends to a constant value. This behavior is typical for massive particles (hydrogen clouds) outside galaxies. Thus the rotational galactic curves can be naturally explained in brane world models. The galaxy is embedded in a modified, spherically symmetric geometry, generated by the nonzero contribution of the Weyl tensor from the bulk. The extra terms, which can be described in terms of a dark radiation term $U$ and a dark pressure term $P$, act as a "matter" distribution outside the galaxy. The particles moving in this geometry feel the gravitational effects of $U$ and $P$, which can also be described, equivalently, by means of a mass term.

The behavior of the metric coefficients and of the angular velocity in the solutions we have obtained depend on two arbitrary constants of integration $k$ and $B$. Their numerical value can be obtained by assuming the continuity of the metric coefficient $\exp (\lambda)$ across the vacuum boundary of the galaxy. For simplicity we assume that inside the "normal" (baryonic) luminous matter, with density $\rho_{B}$, which form a galaxy, the nonlocal effects of the Weyl tensor can be neglected. We define the vacuum boundary $r_{0}$ of the galaxy (which for simplicity is assumed to have spherical symmetry) by the condition $\rho_{B}\left(r_{0}\right) \approx 0$. Therefore at the vacuum boundary the metric coefficient $\exp (\lambda)=1-2 G M_{B} / r_{0}$, where $M_{B}=4 \pi \int_{0}^{r_{0}} \rho_{B}(r) r^{2} d r$ is the total baryonic mass inside the radius $r_{0}$. The continuity of $\exp (\lambda)$ through the surface $r$ $=r_{0}$ gives

$$
1-\frac{2 G M_{B}}{r_{0}}=\frac{\psi^{2}\left(r_{0}\right)}{B^{2}}=\frac{k^{2}}{B^{4}} \frac{1}{\left[1-v_{t g}^{2}\left(r_{0}\right)\right]^{2}},
$$

leading to

$$
\frac{k^{2}}{B^{4}}=\left(1-\frac{2 G M_{B}}{r_{0}}\right)\left[1-v_{t g}^{2}\left(r_{0}\right)\right]^{2} .
$$

Therefore the ratio $k^{2} / B^{4}$ can be determined observationally. With the help of Eq. (59) the limiting angular velocity of the test particle rotating in the conformally symmetric gravitational field on the brane, given by Eq. (53), can be expressed, as a function of the total baryonic mass of the galaxy only, in the form

$$
v_{t g \infty}=\sqrt{1-\frac{6 \sqrt{1-\frac{2 G M_{B}}{r_{0}}}\left(1-\frac{G M_{B}}{r_{0}}\right)}{3 \sqrt{1-\frac{2 G M_{B}}{r_{0}}}\left(1-\frac{G M_{B}}{r_{0}}\right)+\sqrt{12-3\left(1-\frac{2 G M_{B}}{r_{0}}\right)\left(1-\frac{G M_{B}}{r_{0}}\right)^{2}}}},
$$

where we have also used the Newtonian approximation $v_{t g}^{2}\left(r_{0}\right)=G M_{B} / r_{0}$ to eliminate the angular velocity of a test particle at the vacuum boundary of the galaxy.

Since for a galaxy $G M_{B} / r_{0}$ has a very small value, we can expand $v_{t g o \infty}$ in a power series of $G M_{B} / r_{0}$, thus obtaining

$$
v_{t g \infty} \approx \frac{2}{\sqrt{3}} \sqrt{\frac{G M_{B}}{r_{0}}}+\frac{1}{12 \sqrt{3}}\left(\frac{G M_{B}}{r_{0}}\right)^{3 / 2}+O\left[\left(\frac{G M_{B}}{r_{0}}\right)^{5 / 2}\right] .
$$

For a galaxy with baryonic mass of the order $10^{9} M_{\odot}$ and radius of the order of $r_{0} \approx 70 \mathrm{kpc}$, Eq. (61) gives $v_{t g}$ $\approx 287 \mathrm{~km} / \mathrm{s}$, which is of the same order of magnitude as the observed value of the angular velocity of the galactic rotation curves.

From the field equation (8) it follows that in the vacuum outside the galaxy the metric tensor component $\exp (-\lambda)$ can be expressed in terms of the dark radiation only as exp $(-\lambda)=1-2 G M_{U} / r$, where $M_{U}=3 \alpha \int_{r_{0}}^{r} U(r) r^{2} d r$ represents the "mass" associated with the dark radiation component of the energy-momentum tensor on the brane. By using the conformal symmetry and the expression of the ratio $k^{2} / B^{4}$ we obtain for $M_{U}$ the expression

$$
M_{U}(r)=\frac{r}{2 G}\left\{1-\left(1-\frac{2 G M_{B}}{r_{0}}\right)\left[\frac{1-v_{t g}^{2}\left(r_{0}\right)}{1-v_{t g}^{2}(r)}\right]^{2}\right\} .
$$

Since $v_{t g}^{2}\left(r_{0}\right)$ and $v_{t g}^{2}(r)$ are much smaller than 1 , it follows that the dark radiation mass can be approximated by the very simple scaling relation

$$
M_{U}(r) \approx M_{B} \frac{r}{r_{0}} .
$$

$M_{U}$ is linearly increasing with distance and is proportional to the baryonic mass of the galaxy. In the Newtonian limit, from the equality between the centrifugal force and the gravitational force it follows that $M_{B} / r_{0}=v_{t g}^{2}\left(r_{0}\right) / G$, leading to

$$
M_{U}(r) \approx \frac{v_{t g}^{2}\left(r_{0}\right)}{G} r
$$


In conclusion, in the present paper we have investigated conformally symmetric vacuum solutions of the gravitational field equations on the brane and analyzed the motion of test particles in stable circular orbits in this geometry. By using the continuity of the metric coefficients a complete description of the motion of the particles outside a galaxy can be obtained. In the large distance limit the angular velocity of the particles tends to a constant value, which can be determined as a function of the baryonic (luminous) mass and the radius of the galaxy. All the relevant physical quantities, including the dark energy and the dark pressure terms, which describe the nonlocal effects due to the gravitational field of the bulk, are expressed in terms of observable parameters. More general conformally symmetric solutions on the brane, and their physical properties, will be considered in detail in a future publication.

\section{APPENDIX}

In this appendix we present the values of the Kretschmann scalars RiemSq $\equiv R_{i j k l} R^{i j k l}$ and RicciSq $\equiv R_{i j} R^{i j}$ and some values of the independent set of the scalar polynomial invariants $\left\{R, r_{1}, r_{2}, w_{2}\right\}$ for the exact static, spherically symmetric vacuum brane with conformal symmetry for some particular values of the integration constants $k$ and $B$.

For $k=2$ and $B=1$ the expressions of the invariants are

$$
\begin{gathered}
R=0, \quad \text { RicciSq }=18 R_{0}^{-4} e^{-4 /(r-1)}(r-1)^{4}\left(9-8 r+12 r^{2}\right. \\
\left.-16 r^{3}+6 r^{4}\right), \\
\text { RiemSq }=72 R_{0}^{-4} e^{-4 /(r-1)}(r-1)^{4}\left(6-4 r+6 r^{2}-8 r^{3}+3 r^{4}\right), \\
\text { (A2) }
\end{gathered}
$$

For $B=1$ and $k= \pm 1$ we obtain

$$
R=0, \quad \text { RicciSq }=\frac{4 R_{0}^{2}\left(6 r^{2} \pm 8 R_{0} r+3 R_{0}^{2}\right)}{r^{8}},
$$

$$
\begin{gathered}
\text { RiemSq }=2 \text { RicciSq, } \\
r_{1}=\frac{\text { RicciSq }}{4}, \quad r_{2}=\frac{3 R_{0}^{3}\left(2 r^{3} \pm 5 R_{0} r^{2}+4 R_{0}^{2} r \pm R_{0}^{3}\right)}{r^{12}}, \\
\operatorname{Re}\left(w_{2}\right)=0 .
\end{gathered}
$$

[1] C.M. Will, Theory and Experiment in Gravitational Physics (Cambridge University Press, Cambridge, England, 1993).

[2] J. Binney and S. Tremaine, Galactic Dynamics (Princeton University Press, Princeton, 1987); M. Persic, P. Salucci, and F. Stel, Mon. Not. R. Astron. Soc. 281, 27 (1996); A. Boriello and P. Salucci, ibid. 323, 285 (2001).

[3] I.F.M. Albuquerque and L. Baudis, Phys. Rev. Lett. 90, 221301 (2003).

[4] T. Matos and F.S. Guzman, Class. Quantum Grav. 18, 5055 (2001); L.G. Cabral-Rosetti, T. Matos, D. Nuez, and R.A. Sussman, ibid. 19, 3603 (2002); J.E. Lidsey, T. Matos, and L. Arturo Urena-Lopez, Phys. Rev. D 66, 023514 (2002); T. Matos and D. Nunez, astro-ph/0303594.

[5] K. Lake, Phys. Rev. Lett. 92, 051101 (2004).

[6] S. Bharadwaj and S. Kar, Phys. Rev. D 68, 023516 (2003).

[7] R.H. Sanders, Astron. Astrophys. 136, L21 (1984); 154, 135 (1986).

[8] M. Milgrom, Astrophys. J. 270, 365 (1983); J. Bekenstein and M. Milgrom, ibid. 286, 7 (1984); M. Milgrom, New Astron. Rev. 46, 741 (2002); Astrophys. J. Lett. 599, L25 (2003).

[9] P.D. Mannheim, Astrophys. J. 419, 150 (1993); 479, 659 (1997).

[10] J.W. Moffat and I.Y. Sokolov, Phys. Lett. B 378, 59 (1996).

[11] V.V. Zhytnikov and J.M. Nester, Phys. Rev. Lett. 73, 2950 (1994).

[12] M. Pavsic and V. Tapia, "Resource Letter on geometrical results for Embeddings and Branes," gr-qc/0010045.

[13] L.P. Eisenhart, Riemannian Geometry (Princeton University Press, Princeton, 1949).
[14] I. Robinson and Y. Ne'eman, Rev. Mod. Phys. 37, 201 (1965).

[15] K. Akama, in Gauge Theory and Gravitation, Proceedings, Nara, 1982, edited by K. Kikkawa, N. Nakanishi, and H. Nariai, Lecture Notes in Physics Vol. 176 (Springer-Verlag, Berlin, 1983), p. 267.

[16] V.A. Rubakov and M.E. Shaposhnikov, Phys. Lett. 125B, 136 (1983).

[17] I.A. Bandos and W. Kummer, Int. J. Mod. Phys. A 14, 4881 (1999).

[18] L. Randall and R. Sundrum, Phys. Rev. Lett. 83, 3370 (1999); 83, 4690 (1999).

[19] R. Maartens, Living Rev. Relativ. 7, 1 (2004).

[20] T. Shiromizu, K. Maeda, and M. Sasaki, Phys. Rev. D 62, 024012 (2000); M. Sasaki, T. Shiromizu, and K. Maeda, ibid. 62, 024008 (2000).

[21] K. Maeda and D. Wands, Phys. Rev. D 62, 124009 (2000); R. Maartens, ibid. 62, 084023 (2000); A. Campos and C.F. Sopuerta, ibid. 63, 104012 (2001); 64, 104011 (2001); C.-M. Chen, T. Harko, and M.K. Mak, ibid. 64, 044013 (2001); D. Langlois, Phys. Rev. Lett. 86, 2212 (2001); C.-M. Chen, T. Harko, and M.K. Mak, Phys. Rev. D 64, 124017 (2001); L. Anchordoqui, J. Edelstein, C. Nunez, S.P. Bergliaffa, M. Schvellinger, M. Trobo, and F. Zyserman, ibid. 64, 084027 (2001); A. Coley, ibid. 66, 023512 (2002); A. Coley, Class. Quantum Grav. 19, L45 (2002); J.D. Barrow and R. Maartens, Phys. Lett. B 532, 153 (2002); H. Kudoh and T. Tanaka, Phys. Rev. D 65, 104034 (2002); H.A. Bridgman, K.A. Malik, and D. Wands, ibid. 65, 043502 (2002); C.-M. Chen, T. Harko, W.F. Kao, and M.K. 
Mak, Nucl. Phys. B636, 159 (2002); M. Szydlowski, M.P. Dabrowski, and A. Krawiec, Phys. Rev. D 66, 064003 (2002); T. Harko and M.K. Mak, Class. Quantum Grav. 20, 407 (2003); C.-M. Chen, T. Harko, W.F. Kao, and M.K. Mak, J. Cosmol. Astropart. Physics 11, 005 (2003); I. Brevik, K. Børkje, and J.P. Morten, gr-qc/0310103; T. Harko and M.K. Mak, Class. Quantum Grav. 21, 1489 (2004).

[22] N. Dadhich and S.G. Ghosh, Phys. Lett. B 518, 1 (2001); M.G. Santos, F. Vernizzi, and P.G. Ferreira, Phys. Rev. D 64, 063506 (2001); M. Bruni, C. Germani, and R. Maartens, Phys. Rev. Lett. 87, 231302 (2001); H.-C. Kim, S.-H. Moon, and J.H. Yee, J. High Energy Phys. 02, 046 (2002); M. Govender and N. Dadhich, Phys. Lett. B 538, 233 (2002); T. Wiseman, Class. Quantum Grav. 19, 3083 (2002); R. Neves and C. Vaz, Phys. Rev. D 66, 124002 (2002); L.A. Anchordoqui, H. Goldberg, and A.D. Shapere, ibid. 66, 024033 (2002); H. Kudoh, T. Tanaka, and T. Nakamura, ibid. 68, 024035 (2003).
[23] N. Dadhich, R. Maartens, P. Papadopoulos, and V. Rezania, Phys. Lett. B 487, 1 (2000).

[24] C. Germani and R. Maartens, Phys. Rev. D 64, 124010 (2001).

[25] R. Casadio, A. Fabbri, and L. Mazzacurati, Phys. Rev. D 65, 084040 (2002); S. Shankaranarayanan and N. Dadhich, Int. J. Mod. Phys. D (to be published), gr-qc/0306111; M. Visser and D.L. Wiltshire, Phys. Rev. D 67, 104004 (2003); R. Casadio and L. Mazzacurati, Mod. Phys. Lett. A 18, 651 (2003).

[26] T. Harko and M.K. Mak, Phys. Rev. D 69, 064020 (2004).

[27] L. Herrera and J. Ponce de Leon, J. Math. Phys. 26, 2302 (1985); 26, 2018 (1985); 26, 778 (1985).

[28] M.K. Mak and T. Harko, Int. J. Mod. Phys. D 13, 149 (2004).

[29] R. Maartens and M.S. Maharaj, J. Math. Phys. 31, 151 (1990).

[30] K. Santusuosso, D. Pollney, N. Pelavas, P. Musgrave, and K. Lake, Comput. Phys. Commun. 115, 381 (1998).

[31] E. Zakhary and J. Carminati, J. Math. Phys. 42, 1474 (2001); J. Carminati, E. Zakhary, and R.G. McLenaghan, ibid. 43, 492 (2002). 